\title{
Endovascular laser treatment of varicose veins of the lower limbs: results and personal experience
}

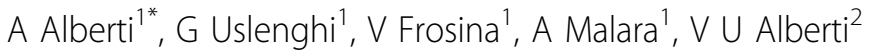 \\ From de Senectute: Age and Health Forum \\ Catanzaro, Italy. 5-7 December 2009
}

\section{Background}

Great saphenous vein (GSV) reflux is an important component of the pathophysiology of primary venous insufficiency. For nearly a century vein saphenous stripping has remained the standard surgical therapy with the recurrence rates from $20 \%$ to $80 \%$.

Endovenous laser therapy (EVLT) is an alternative and minimally invasive procedure for the treatment of saphenous vein incompetence.

This procedure is designed to ablate GSV as a percutaneous approach to minimize the complications and discomfort associated with surgical stripping.

We have performed the procedure at the Operative Unit of Vascular Surgery - General Hospital of Reggio Calabria, had compared the traditional surgery with EVLT in the management of the saphenous reflux.

\section{Materials and methods}

All patients were selected from our Division of Vascular Surgery, history and physical examination were performed. Duplex color scanning to document patency of deep veins and to evaluate to valve reflux time.

Clinical records of 78 consecutive patients who underwent endovenous GSV ablation with EVLT over a 5 year period between January 2004 and June 2008, were retrospectively reviewed.

All patients had symptomatic varicose veins with documented GSV incompetence and were classified to the CEAP classification.

Under ultrasound guidance the GSV was punctured with an $18-19 \mathrm{G}$ needle and was after cannulated 45 $\mathrm{cm}$ angio-shaeath over a J - tip guidewire. And $810 \mathrm{~nm}$ diode laser fiber.

Early post-operative evaluation by-color-duplex-scanning was started1 month from EVLT, successful

${ }^{1}$ Department of Surgery, Operative Unit of Vascular and Endovascular Surgery, Riuniti Hospital City of Reggio Calabria, 89124, Italy obliteration was confirmed by the evidence of a non compressible GSV and no color flow on duplex analysis.

\section{Results}

Ninety-six limbs in 78 patients were treated, $84 \%$ woman and $16 \%$ man.

Mean age of 53 (range $25-84$ ). All patients had symptomatic varicose vein and primary valvular incompetence. Adjunctive procedures included phlebectomies and subfascial endoscopic perforator surgery (8 patients).

Immediate success was obtained in all patients (100\%). In no case the procedure was aborted.

Early post-operative duplex color revealed 4/78 early GSV recanalization, 2 partial and 2 total.

No deep venous thrombosis were reveled.

Late post-operative color Doppler and clinical examination are detected in the $8(9 \%)$ patients symptomatic leg varicosities and duplex ultrasound reflux in GSV.

\section{Conclusions}

Endovascular laser treatment of GSV incompetence have been introduced as minimal alternatives to open surgical ligation and stripping. Although the surgical stripping can lead to painful and prolonged postoperative recovery with risk of infection, hematoma, and nerve lesions.

\section{Author details}

${ }^{1}$ Department of Surgery, Operative Unit of Vascular and Endovascular Surgery, Riuniti Hospital City of Reggio Calabria, 89124, Italy. ${ }^{2}$ Department of Cardiovascular Surgery, Operative Unit of Vascular Surgery, "San Filippo Neri" Hospital, City of Rome, 00135, Italy.

Published: 19 May 2010

doi:10.1186/1471-2318-10-S1-A41

Cite this article as: Alberti et al:: Endovascular laser treatment of varicose veins of the lower limbs: results and personal experience. $B M C$ Geriatrics 2010 10(Suppl 1):A41. 